COMMENTARY

\title{
Continuous glucose monitoring: coming of age?
}

\author{
J Hans DeVries \\ Departments of Internal Medicine and Endocrinology and Metabolism, Academic Medical Center, University of Amsterdam, 1105 AZ Amsterdam, \\ The Netherlands \\ (Correspondence should be addressed to J H DeVries; Email: j.h.devries@amc.uva.nl)
}

\begin{abstract}
In a recent issue of the Journal of Clinical Endocrinology and Metabolism, an Endocrine Society Clinical Practice Guideline on Continuous Glucose Monitoring (CGM) was published, co-sponsored by the European Society of Endocrinology. Such a guideline is a necessary step in the maturation of any new technology. This commentary tries to delineate where we stand some 10 years after the first retrospective CGM device entered the market from a European perspective.
\end{abstract}

European Journal of Endocrinology 166 1-4

\section{Introduction}

The Endocrine Society appointed a Task Force to formulate practice guidelines to identify settings in which patients are most likely to benefit from continuous glucose monitoring (CGM) (1). The Task Force evaluated three potential uses of CGM: i) real-time CGM (RT-CGM) in adult hospital settings; ii) RT-CGM in children and adolescent outpatients and iii) RT-CGM in adult outpatients. Overall, it gives an excellent overview of the current status, and makes worthwhile reading (1).

\section{Hospital setting}

The Task Force recommends against the use of RT-CGM alone for glucose management in the intensive care unit (ICU) or operating room until further studies provide sufficient evidence for its accuracy and safety in these settings. The main concern here is the limited number and size of studies on sensor accuracy, with very little data on the hypoglycaemic range. Hypoglycaemia has been noted as a risk factor for mortality in the ICU setting (2). Furthermore, there is conflicting evidence on possible disturbing factors such as hypotension, vasopressor medication, etc. The Task Force extensively discusses the recent controversy arisen around the use of point of care glucose measurements in the ICU. It also rightfully notes the need for re-establishing the efficacy of intensive insulin therapy in achieving tight glycaemic control, following the unexpected increased mortality noted in NICE-SUGAR (3). Of note, the first study reporting on glycaemic outcomes in the ICU, showing a lower incidence of hypoglycaemia with the use of CGM, is not discussed (4).

\section{Outpatient use}

As to CGM in the outpatient setting, the Task Force recommends its use in patients from all age groups above 8 years old, either to lower $\mathrm{HbA1c}$ or to maintain a low $\mathrm{HbA1c}$ while lowering the incidence of non-severe hypoglycaemia. The guideline makes a distinction between children and adolescents on the one hand and adults on the other hand. This distinction also follows from the results of a landmark trial in this area, the Juvenile Diabetes Research Foundation CGM trial (5). In fact this trial consists of three identical parallel trials, one in children, one in adolescents and one in adults. At first glance, only adults seemed to benefit from CGM, with a significant $0.5 \% \mathrm{HbA} 1 \mathrm{c}$ decrease, compared with the control group using self-monitoring of blood glucose (SMBG) only. At closer examination, this unexpected and perhaps even disappointing outcome could readily be explained by the large proportion of participants discontinuing CGM use. In a per protocol analysis, those using the device benefited to a similar extent, irrespective of their age. Thus, the following truism emerged: a device that is not used will not work. However, the fact that many users, i.e. motivated trial participants, discontinued the use of CGM illustrates that this is not a magic bullet. Few relevant predictors of use could be identified. It is obvious that user friendliness, including the high rate of false-positive alarms, needs improvement. Also, it seems obvious that many people choose to ignore their disease as much as possible and live their life as if the disease was not there, probably for right personal reasons (6).

This brings us to the question on how appropriate the intention-to-treat analysis is in this field. Intention-to-treat 
analysis reached a sacrosanct status due to its validity in drug trials, especially pertaining to trials evaluating drugs prescribed to reduce a given risk. In conditions such as hypertension and osteoporosis, roughly half of those who are prescribed a drug stop it in the first year, as determined from pharmacy databases. On top of this, there are people who pick up their prescription, but do not take all the tablets, or any tablet for that matter. Rightfully, third-party payers (publicly or privately funded healthcare systems) argue that all pills, including those not swallowed, have to be paid. Therefore the drug should have such a powerful effect that this remains evident as patients who were prescribed the drug but do not take it, for any reason, are still included in the efficacy analysis. Thus, the intention-to-treat analysis was born. However, now returning to the CGM field, it seems valid to take the per protocol analysis as the primary analysis. If CGM devices are not used they do not need to be paid for, and the number of sensors used is readily determined by the treatment team by taking a look at the sensor results uploaded to a website maintained by the manufacturer. However, the third-party payers will rightfully ask for cost-efficacy analyses. Only one is known to me, which is not mentioned by the guideline (7). This analysis reports on cost-efficacy in the JDRF CGM trial, using the intention-to-treat results. They estimated a price of USD 100000 for one QALY gained, above the cut off level usually considered justified for new drugs or technology.

CGM was brought to the market in 1999. If one would have polled diabetes healthcare providers on their expectations at that time, a reduction in the occurrence of severe hypoglycaemia would have been mentioned by most, if not all. Disappointingly, such a reduction was not seen, one trial even noting an increase in the occurrence of severe hypoglycaemia (8). Reasons for this include the fact that the sound and vibration alarms are insufficiently able to alert the user during hypoglycaemia, when cognitive dysfunction has already set in (9). Secondly, devices are likely to be taken off during activities that carry a high risk to induce hypoglycaemia, such as full contact sports. Thirdly, despite ongoing improvement, accuracy of current glucose sensors is still suboptimal, with a relatively large number of both false-positive and false-negative alarms (10). To improve the positive predictive value of the hypoglycaemia alarm, many patients choose to set their individual threshold for the hypoglycaemia alarm at lower values, thereby by definition also increasing the false-negative rate. The Task Force notes a reduction in the occurrence of severe hypoglycaemia during observational follow-up studies of the JDRF CGM trial. Possibly the learning curve to prevent severe hypoglycaemia by these devices takes longer than expected. These uncontrolled observations raise hopes, but will not be convincing for third-party payers. Controlled trials in people at high risk for severe hypoglycaemia, notably those with hypoglycaemia unawareness, are urgently needed.
The Task Force does not mention whether the method of insulin administration (i.e. insulin pumps vs multiple daily injections (MDI)) should influence whether RT-CGM is supported. In adults, however, it should be noted that in all published studies $(5,9,11-15) 30$ patients were randomised to CGM use in combination with MDI therapy. Also, the Task Force abstains from commenting on the two trials showing HbAlc improvements of $0.6-1.2 \%$ when using a combined insulin pump-CGM device with mealbolus advisor compared with MDI with SMBG $(16,17)$. Indeed, probably this combined device should be seen as a single integrated device, rather than as a combination of two devices with a bolus advisor.

The Task Force suggests that treatment guidelines be provided to patients to allow them to safely and effectively take advantage of the information provided to them by RT-CGM. Will guidelines for how to optimally use these devices be useful? Intuitively one would expect this, but it should be noted that the most successful trials in this field just handed out the device without any education, if only because the healthcare providers were at the beginning of their personal learning curve $(5,12)$.

The Task Force suggests that short-term retrospective CGM may be of benefit in adult patients with diabetes to detect nocturnal hypoglycaemia, the dawn phenomenon, and postprandial hyperglycaemia, and to assist in the management of hypoglycaemic unawareness and when significant changes are made to their diabetes regimen. The Task Force notes some small trials that showed benefit, but ignores the largest trial on retrospective CGM (18), perhaps because results of this trial were not reported separately for people with type 1 and type 2 diabetes.

\section{Pregnancy}

The Task Force chose not to comment on one of the most frequently mentioned indications for CGM, that is pregnancy. Based on one small but high-quality trial, the use of intermittent retrospective CGM during pregnancy can be supported (19). The lack of trials in pregnancy reminds us of a similar ill foundation for the widely claimed benefits of insulin pump therapy during pregnancy. Similarly instructive is the fact that the first randomised trial of rapid-acting insulin analogues in pregnant women with type 1 diabetes was only published more than 10 years after the introduction of this drug class (20).

\section{CGM Reimbursement in Europe}

How applicable is this guideline for Europe? It seems likely it will give further fuel to the many ongoing debates with third-party payers throughout Europe. Current status of reimbursement in Europe is as 
follows. In Israel, children with type 1 diabetes who have experienced more than two severe hypoglycaemic episodes within 1 year are entitled to CGM compensation. In The Netherlands and part of Italy, retrospective CGM is currently reimbursed. The Czech Republic covers up to four sensors per year for retrospective CGM and in Sweden, RT-CGM is reimbursed for patients using CSII and experiencing two or more severe hypoglycaemic episodes per year, children who require at least ten plasma glucose tests per $24 \mathrm{~h}$ and patients with $\mathrm{HbA1c}>10 \%$ while receiving optimised insulin therapy. In the Netherlands, reimbursement was recently given for adults with an $\mathrm{HbA1c}$ persistently above $8 \%$ despite intensive treatment, children and pregnant women, all with type 1 diabetes. In addition, RT-CGM is now covered by the majority of health plans in the USA, including the federal Medicare programme. Reimbursement is generally available for type 1 patients with severe hypoglycaemia or those who are not meeting American Diabetes Association HbA1c targets (21).

\section{Conclusion}

CGM is coming of age. If reimbursement follows the published evidence, increasing reimbursement is expected for pump patients who do not succeed in maintaining a satisfactory $\mathrm{HbA1c}$, but who show that they can sustain CGM use for a certain period of time. Clinical trials in patients with hypoglycaemia unawareness are urgently needed.

\section{Declaration of interest}

$\mathrm{J} H \mathrm{DeVries}$ or institutions he is associated with have received speaker fees and/or research support from and Abbott Diabetes Care, Dexcom, Inc. and Medtronic.

\section{Funding}

This research did not receive any specific grant from any funding agency in the public, commercial or not-for-profit sector.

\section{References}

1 Klonoff DC, Buckingham B, Christiansen JS, Montori VM, Tamborlane WV, Vigersky RA \& Wolpert H. Continuous glucose monitoring: an Endocrine Society Clinical Practice Guideline. Journal of Clinical Endocrinology and Metabolism 201196 2968-2979. (doi:10.1210/jc.2010-2756)

2 Hermanides J, Bosman RJ, Vriesendorp TM, Dotsch R, Rosendaal FR, Zandstra DF, Hoekstra JB \& DeVries JH. Hypoglycemia is associated with intensive care unit mortality. Critical Care Medicine 201038 1430-1434. (doi:10.1097/CCM. Ob013e3181de562c)

3 Finfer S, Chittock DR, Su SY, Blair D, Foster D, Dhingra V, Bellomo R, Cook D, Dodek P, Henderson WR, Hébert PC, Heritier S, Heyland DK, McArthur C, McDonald E, Mitchell I, Myburgh JA, Norton R, Potter J, Robinson BG \& Ronco JJ. Intensive versus conventional glucose control in critically ill patients. New England Journal of Medicine 2009360 1283-1297. (doi:10.1056/NEJM oa0810625)

4 Holzinger U, Warszawska J, Kitzberger R, Wewalka M, Miehsler W, Herkner H \& Madl C. Real-time continuous glucose monitoring in critically ill patients: a prospective randomized trial. Diabetes Care 201033 467-472. (doi:10.2337/dc09-1352)

5 Tamborlane WV, Beck RW, Bode BW, Buckingham B, Chase HP, Clemons R, Fiallo-Scharer R, Fox LA, Gilliam LK, Hirsch IB, Huang ES, Kollman C, Kowalski AJ, Laffel L, Lawrence JM, Lee J, Mauras N, O'Grady M, Ruedy KJ, Tansey M, Tsalikian E, Weinzimer S, Wilson DM, Wolpert $\mathrm{H}$, Wysocki $\mathrm{T} \&$ Xing D. Continuous glucose monitoring and intensive treatment of type 1 diabetes. New England Journal of Medicine 2008359 1464-1476. (doi:10.1056/NEJMoa0805017)

6 DeVries JH, Snoek FJ \& Heine RJ. Persistent poor glycaemic control in adult type 1 diabetes. A closer look at the problem. Diabetic Medicine 200421 1263-1268. (doi:10.1111/j.1464-5491.2004. 01386.x)

7 Huang ES, O'Grady M, Basu A, Winn A, John P, Lee J, Meltzer D, Kollman C, Laffel L, Tamborlane W, Weinzimer S \& Wysocki T \& Juvenile Diabetes Research Foundation Continuous Glucose Monitoring Study Group. The cost-effectiveness of continuous glucose monitoring in type 1 diabetes. Diabetes Care 201033 1269-1274. (doi:10.2337/dc09-2042)

8 Hirsch IB, Abelseth J, Bode BW, Fischer JS, Kaufman FR, Mastrototaro J, Parkin CG, Wolpert HA \& Buckingham BA. Sensor-augmented insulin pump therapy: results of the first randomized treat-to-target study. Diabetes Technology \& Therapeutics 200810 377-383. (doi:10.1089/dia.2008.0068)

9 Buckingham B, Wilson DM, Lecher T, Hanas R, Kaiserman K \& Cameron F. Duration of nocturnal hypoglycemia before seizures. Diabetes Care 200831 2110-2112. (doi:10.2337/dc08-0863)

10 Wentholt IM, Hoekstra JB \& DeVries JH. Continuous glucose monitors: the long-awaited watch dogs? Diabetes Technology $\mathcal{E}$ Therapeutics 20079 399-409. (doi:10.1089/dia.2007.0215)

11 Peyrot M \& Rubin RR. Patient-reported outcomes for an integrated real-time continuous glucose monitoring/insulin pump system. Diabetes Technology \& Therapeutics 200911 57-62. (doi:10.1089/ dia.2008.0002)

12 Deiss D, Bolinder J, Riveline JP, Battelino T, Bosi E, Tubiana-Rufi N, Kerr D \& Phillip M. Improved glycemic control in poorly controlled patients with type 1 diabetes using real-time continuous glucose monitoring. Diabetes Care 200629 2730-2732. (doi:10.2337/ dc06-1134)

13 Raccah D, Sulmont V, Reznik Y, Guerci B, Renard E, Hanaire H, Jeandidier N \& Nicolino M. Incremental value of continuous glucose monitoring when starting pump therapy in patients with poorly controlled type 1 diabetes: the RealTrend study. Diabetes Care 200932 2245-2250. (doi:10.2337/dc09-0750)

14 O'Connell MA, Donath S, O'Neal DN, Colman PG, Ambler GR, Jones TW, Davis EA \& Cameron FJ. Glycaemic impact of patient-led use of sensor-guided pump therapy in type 1 diabetes: a randomised controlled trial. Diabetologia 200952 1250-1257. (doi:10.1007/s00125-009-1365-0)

15 Juvenile Diabetes Research Foundation Continuous Glucose Monitoring Study Group. The effect of continuous glucose monitoring in well-controlled type 1 diabetes. Diabetes Care 200932 1378-1383. (doi:10.2337/dc09-0108)

16 Bergenstal RM, Tamborlane WV, Ahmann A, Buse JB, Dailey G, Davis SN, Joyce C, Peoples T, Perkins BA, Welsh JB, Willi SM \& Wood MA \& STAR 3 Study Group. Effectiveness of sensoraugmented insulin-pump therapy in type 1 diabetes. New England Journal of Medicine 2010363 311-320. (doi:10.1056/ NEJMoa1002853)

17 Hermanides J, Nørgaard K, Bruttomesso D, Mathieu C, Frid A, Dayan CM, Diem P, Fermon C, Wentholt IME, Hoekstra JBL \& DeVries JH. Sensor augmented pump therapy lowers HbAlc in suboptimally controlled type 1 diabetes: a randomised controlled trial. Diabetic Medicine 201128 1158-1167. (doi:10.1111/j. 1464-5491.2011.03256.x) 
18 Cooke D, Hurel SJ, Casbard A, Steed L, Walker S, Meredith S, Nunn AJ, Manca A, Sculpher M, Barnard M, Kerr D, Weaver JU, Ahlquist J \& Newman SP. Randomized controlled trial to assess the impact of continuous glucose monitoring on $\mathrm{HbA}(1 \mathrm{c})$ in insulintreated diabetes (MITRE Study). Diabetic Medicine 200926 540-547. (doi:10.1111/j.1464-5491.2009.02723.x)

19 Murphy HR, Rayman G, Lewis K, Kelly S, Johal B, Duffield K, Fowler D, Campbell PJ \& Temple RC. Effectiveness of continuous glucose monitoring in pregnant women with diabetes: randomised clinical trial. BMJ 2008337 a1680. (doi:10.1136/bmj.a1680)

20 Mathiesen ER, Kinsley B, Amiel SA, Heller S, McCance D, Duran S, Bellaire S \& Raben A \& Insulin Aspart Pregnancy Study Group.
Maternal glycemic control and hypoglycemia in type 1 diabetic pregnancy: a randomized trial of insulin aspart versus human insulin in 322 pregnant women. Diabetes Care 200730 771-776. (doi:10.2337/dc06-1887)

21 Hermanides J \& DeVries JH. Sense and nonsense in sensors. Diabetologia 201053 593-596. (doi:10.1007/s00125-009-1649-4)

Received 23 October 2011

Accepted 3 November 2011 